\title{
Cognitive conflict as a tool of overcoming obstacles in understanding infinity
}

\author{
Jiří Cihlář, Petr Eisenmann, Magdalena Krátká and Petr Vopěnka
}

\begin{abstract}
This paper, based on current research, is devoted to obstacles that appear in the process of understanding the concept of infinity. In its introductory part the fundamental types of epistemology obstacles are formulated. The cognitive conflict and its role in overcoming these obstacles are analysed in the following section of this research. Finally, the authors focus on research methodology and the results of the three-year research project. The problems are illustrated by means of real experimental interviews.
\end{abstract}

Key words and phrases: obstacle, concept of infinity, cognitive conflict.

ZDM Subject Classification: D70.

\section{Introduction}

The concept of infinity is a key concept in mathematics and its teaching. In the history of mathematics the development of knowledge about infinity creates crucial milestones important for its further development. If we accept the hypothesis of the so called genetic parallel, which means that the ontogenetic development is not independent of the phylogenetic development, it is possible to assume that the obstacles we find in the phylogeny of the concept of infinity, can be identified also in the ontogeny and that the overcoming of these obstacles is a necessary component of the cognitive process of an individual. Understanding of 


$$
\text { "tmcs-eisenmann-etal" — 2009/11/4 — 11:03 — page 280 — \#2 }
$$

many concepts which pupils meet during their learning process is dependent on the gradual concept of the building of infinity (point, line, line segment, decimal notation of numbers etc.). We could explain students' understanding by help of Gray and Tall's theory of procept (Gray-Tall [3]), where infinity may be considered as a process (for example the phenomenon 'again and again' or no - end sequence), as a concept (infinity in total), as a procept as well (how we can see in the example of the principle of induction).

At present, within the framework of the three-year GAČR project, our attention is drawn to the study of both the phylogenetic concept development of infinity and to the study of ontogenetic development of the understanding of infinity in our contemporary population. The research should be crowned with a formulation of suggestions of how to overcome the identified obstacles.

The theory of epistemological obstacles is based on the Piaget theory of developmental stages. In this article it is not supposed that the construction of knowledge, as formulated by Piaget and Garcia, is determined only by positive stages of development, but also by means of "negative" stages, including various rules, beliefs and ways of thinking, which form obstacles to the changes leading to further stages (Sierpinska [8]).

We understand an obstacle as a set of knowledge grounded in the knowledge structure of an individual that can be successfully used in one context or situation but in another this set gives wrong results (Brousseau [1]).

On the basis of the authors' experience from the previous research (Krátká [4], Cihlář-Eisenmann-Krátká-Vopěnka [2]) and above all from the research described herein, we have obtained a set of obstacles to understanding infinity and consider them, in principle, as unrecoverable (epistemological):

- Knowledge about "finiteness" (finite sets, finite processes, countability of sets, bounded objects, lengths of line segments, etc.)

- Replacement of an object with its model

- Potential perception of the infinite process

- Knowledge concerning the ordering of a set of natural numbers (an image of the discreet ordering, the atomistic image of the point)

Apart from epistemological obstacles we can also meet the obstacles of a different character i.e. those we could avoid without disturbing the concept-making process. We can certainly include didactic obstacles (for instance experience in spoken language, when the variants of the word "infinity" can be found in the context 


$$
\text { "tmcs-eisenmann-etal" — 2009/11/4 — 11:03 — page } 281 \text { — \#3 }
$$

unacceptable in mathematics, or when it is expected that each task has a singlevalued solution, etc.). Nevertheless, we will not deal with these obstacles in this paper.

In this paper, we elaborate above all the theory of the so called cognitive conflict as a tool for overcoming obstacles in the understanding of infinity.

\section{Cognitive conflict}

The pieces of knowledge which are obstacles that fail in a certain context produce wrong answers. This failure cannot be considered by an individual. It means that he/she does not know that the obtained result or answer is wrong. There must be someone who knows that the answer is wrong. We will talk about an objective observer. It can be an experimenter, teacher or mathematician.

At first the experimenter identifies the contradiction between the answer of an individual and some objective truth (valid in a given mathematical theory), in our case it is the truth valid in school mathematics. It is important to remark that the cause of the same contradiction (i.e. the contradiction manifested in the same way) may be based in different individuals on different obstacles.

If the individual is aware of the contradiction, we talk about the inducing of the cognitive conflict ${ }^{1}$ (CC). The $\mathrm{CC}$ is not a disagreement between a pupil's wrong answer and an objective truth (it is a contradiction), but a conflict between the knowledge producing this wrong answer and the pupil's different knowledge. Therefore the CC is a disagreement in the knowledge structure of an individual. The inducing of the CC means that an individual is aware of the fact that his/her utterances (based on the inconsistence of his/her knowledge structure) are not in accordance. If we use Popper's terminology, the CC occurs in Popper's world 2, and the contradiction in Popper's world 3 (Popper [7]). We should note that not every wrong answer can be brought to the CC. For example, a four to six-year old child thinks that there is a decrease in the amount of the liquid poured from a narrow glass to a wider one. The child evaluates only the final state when the column of the liquid is lower. We cannot talk about the CC, as a child of this age has not created such basic knowledge yet. The argument that it is the same

${ }^{1}$ The conflict is according to Psychological Dictionary "contradiction, disagreement, simultaneous clash of the opposite tendencies, an offer of two or more attractive aims". The cognitive conflict related to infinity is for the first time mentioned by Tall (see Tall [10], [11]). The so called cognitive conflict teaching approach in connection with the comparison of infinite sets is discussed by (see Swan [9]). 


$$
\text { "tmcs-eisenmann-etal" — 2009/11/4 — 11:03 — page } 282 \text { — \#4 }
$$

water, or that nothing has been added or taken (identity), or that it is possible to pour the liquid back to see that nothing has changed (inversion), or that the column is higher but the glass is narrower (reciprocity) can be used successful when talking with children at the age of seven or more, when these preservation operations start to develop gradually (Piaget-Inhelder [6]).

J. Piaget is probably the first to mention the cognitive conflict. He explains the process of learning on the basis of adaptation of the organism to the environment by means of the so called assimilation, when new knowledge falls in the knowledge structure of an individual (this structure can be "only" extended), and accommodation, when for the achievement of any new knowledge it is necessary to rebuild the existing knowledge structures. The process of learning is thus characterised by the constant disturbance of the balance between assimilation and accommodation. When the balance is upset, then the new piece of knowledge cannot be included in the existing cognitive scheme. In that case he speaks about the cognitive conflict, i.e. about the psychological tension and the subsequent effort to solve the problem. The development of intelligence and reasoning is carried out in stages, always on the basis of a certain new situation, which can turnover the current order and thus create the disequilibrium which will have to be compensated by an individual by means of adaptation from which a new, much stronger equilibrium will be created (Piaget [5]).

Tall and Schwarzenberger speak about the so called conscious and subconscious conflicts induced by two "close" (mathematical) mutually irreconcilable concepts. They say that conflicts are induced by the transfer of mathematical ideas into the teaching and learning process when these are inevitably deformed. In a greater detail: in some cases "... the cause of the conflict can be seen to arise from purely linguistic infelicity...", in another case "...the cause of the conflict arises from a genuine mathematical distinction.", and finally "... the conflict arises from particular events in the past experience of an individual pupil." (Tall-Schwarzenberger [12], p. 49). If we compare this with the theory of obstacles, these obstacles are of didactical origin. We assume that the cause of these conflicts can also lie in other types of obstacles, more particularly in epistemological ones. We take into account both source types of the CC. The authors say that if the conflict is conscious we can expect the following reaction: "... the existence of two 'nearby' concepts can cause mental stress arising from the emergence of unstable thoughts" (Tall-Schwarzenberger [12], p. 44). An individual makes an effort to remove this tension, which can be effectively used in the process of overcoming obstacles.
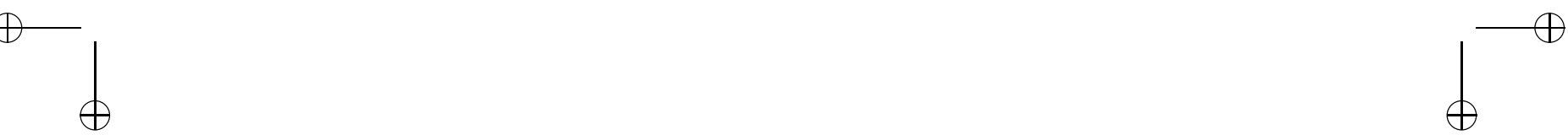


$$
\text { "tmcs-eisenmann-etal" — 2009/11/4 — 11:03 — page } 283 \text { — \#5 }
$$

If an individual faces a cognitive conflict, we distinguish the following possibilities $^{2}$ :

- A vain attempt at removing the conflict: the individual finds out after several trials that he/she has not got sufficient means. The obstacle is so internalised that the individual does not admit that the given piece of knowledge could be the cause of the $\mathrm{CC}$. He/she refuses to remove the $\mathrm{CC}$ and the disagreement in his/her knowledge structure prevails. However, this possibility of facing the $\mathrm{CC}$ is not valueless. It has a propedeutic meaning for other conflicts induced with the aim of overcoming the given obstacle.

- A wrong attempt at removing the conflict: the individual tries to change his/her cognitive structure in a way that he/she adjusts it to the wrong thesis of the conflict. The individual corrects in a wrong way his/her right knowledge. The obstacle does not change or it changes in an undesirable way, and prevails further on. It confirms the presence of the obstacle, for it shows a resistance to conflicts and it changes always as little as possible.

- A successful attempt at removing the conflict: the individual ruins his/her idea about the rightness of the obstacle and changes it in the way that the obstacle produces no more wrong answers in the given context.

By removing the cognitive conflict, the individual gradually overcomes the given obstacle that causes the noticed contradiction and the inducing of the $\mathrm{CC}$. A single obstacle can induce different $\mathrm{CC}$ in various contexts. By removing one $\mathrm{CC}$, the given obstacle is not overcome totally, it is, on the contrary, usually necessary to induce more CC (in different contexts) and to remove them in order to overcome the obstacle.

The cognitive conflict has two functions:

- with the help of the conflict we can identify a particular obstacle (the diagnostic function),

- after the conflict is induced and removed, we can overcome the obstacle and thus we can improve pupils' knowledge structure (the educational function).

A teacher or an experimenter can induce such situations when he/she assumes that an individual gets into the CC. His/her aim is to prove the existence of the assumed obstacles (the diagnostic function) or overcome the assumed obstacle (the educational function).

${ }^{2}$ We should remark that if an individual manages an induced CC it is influenced also by the instant psychosomatic state and by other external conditions, especially by motivation or tiredness. 


$$
\text { "tmcs-eisenmann-etal" — 2009/11/4 — 11:03 — page 284 — \#6 }
$$

A teacher or an experimenter sometimes gets into a situation when a contradiction or the CC appears unexpectedly, or unintentionally. Then the teacher or experimenter should identify if it is caused by an obstacle. If so, the obstacle should be well diagnosed and he should induce other cognitive conflicts in suitable contexts, make pupils remove them and in this way reach the gradual overcoming of the given obstacle.

Our aim is to find and identify obstacles so that teachers noticing a contradiction have a tool for inducing the $\mathrm{CC}$ and in this way they create a situation suitable for the overcoming of this obstacle, as required by Tall a Schwarzenberger: ". . the role of the teacher in finding a suitable resolution will be crucial, and more decisive than such factors as choice of syllabus, textbook...". Unlike them we do not think that the teaching process should be conducted in a way that enables to avoid cognitive conflicts. This is connected with the fact that the authors focus only on the conflicts of didactic obstacles. Like Tsamir or Swan we appreciate their function when creating knowledge (Tsamir [13], Swan [9]) and above all when overcoming obstacles.

The attributes of infinity are mentioned by Tall and Schwarzenberger when they talk about the following (conscious and subconscious) conflict concepts: between decimals and limits, between decimals and fractions, between numbers and limits, and between sequences and series (Tall-Schwarzenberger [12]). As we understand the concept of the $\mathrm{CC}$ in a wider sense, we do not restrict ourselves only to the conflict between two concepts related to the attributes of infinity, but we also observe conflicts between any concept or knowledge, where at least one of them is connected with a determined attribute or observed object.

\section{Research methodology}

The research is conducted at two levels. Firstly, we probe, using a questionnaire, the initial reactions of respondents to identify the key phenomena connected with the creation of ideas of objects introduced in school mathematics related to the concept of infinity (line, point, line segment, plane and its parts, numbers sets). Thus, also with the attributes of infinity. This will lead to more exact specification of the formulation and completion of possible obstacles when building the concept of infinity. From the point of view of this paper about the cognitive conflict as a tool for overcoming obstacles in understanding infinity, the description of this part of research is not so important. 


$$
\text { "tmcs-eisenmann-etal" — 2009/11/4 — 11:03 — page 285 — \#7 }
$$

Secondly, we conduct, using the above mentioned questionnaires, guided experimental interviews with respondents of each age category, also recorded on a camcorder. These recordings are transcribed into protocols after watching. Using the protocols, we set analyses which serve us to elaborate more precise work terminology and to correct the developing theory, which means mainly the formulation of obstacles in understanding infinity and the way of overcoming them. Besides that, we look here for suitable examples of particular illustrations of how to face the cognitive conflict. The experience acquired in the process of the creation and evaluation of the questionnaires helped us significantly to improve the assumptions about the reactions of pupils and students.

\section{Illustration examples}

In the following four examples from real experimental interviews conducted in the year 2007, we illustrate inducing of the cognitive conflicts and make comments on the way pupils faced them. The number in brackets indicates a pause in seconds.

EXAMPLE 1. Jakub, school year 5, very good student

Attribute of boundedness (distinction of bounded, very large and unbounded sets)

On the paper is drawn the following picture (see Figure 1) and the problem is: Is it possible to connect the points $A$ and $B$ with a line so that it does not intersect the straight line $p$ ? (this line does not need to be straight).

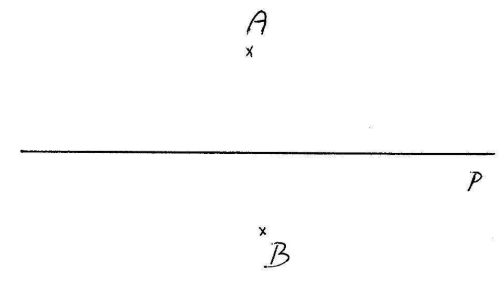

Figure 1

Jakub 43: Yes, it would be possible. (Jakub connects the points $A$ and $B$ with an arch line avoiding the picture of the straight line $p$ - see Figure 2.) 
Exp. 44: Hm. (2) So that the straight line $p$ does not intersect the line $A B$ ?

$\mathrm{J}$ 45: Hm, well, it does not.

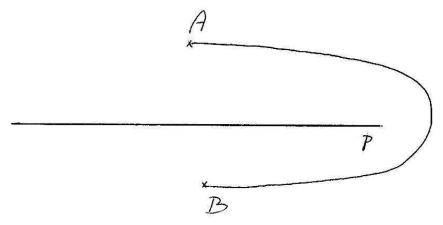

Figure 2

The experimenter notices a contradiction. He thinks that the wrong answer is caused by the fact that Jakub understands a straight line to be its image on the paper, which correlates with the obstacle of the exchange of a geometric object and its model (in this case it is the picture).

E 46: Hm. So I will ask you once again. (The experimenter draws Figure 3.) Here is one straight line $p$ and here is another one - q. Will they intersect?

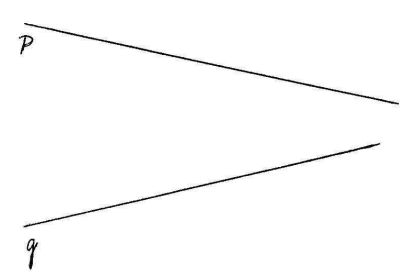

Figure 3

The experimenter tries to induce a cognitive conflict. To do that, he uses an easier task for Jakub without leaving the context. He assumes that Jakub is able to solve the problem correctly.

J 47: Yes. (2) I would find their intersection, if I made them longer. Can I draw it?

E 48: Of course, you can.

J 49: So and so. (Jakub extends the pictures of the straight lines $p$ and $q$ and marks their intersection.)

Jakub succeeded in solving the problem in the given context. However, he is not able to confront his answer with the solution of the previous problem. For this reason, the experimenter makes an explicit reference to the original answer. 


$$
\text { "tmcs-eisenmann-etal" — 2009/11/4 — 11:03 — page } 287 \text { — \#9 }
$$

E 50: OK. Hm. And would it be possible to find an intersection for your line (pointing to the paper) $A B$ and for the straight line as well?

$\mathrm{J}$ 51: I would also make this straight line $p$ longer.

E 52: And can you make it longer?

J 53: (4) Well, it is a straight line, so yes, I can. (Jakub marks the intersection.)

After the repetition of the question ( $\mathrm{E} 50$ ), Jakub is aware of the fact that the same algorithm can also be used here and so he changes his original answer. In a short time period, there is an inducing of the CC as well as its removal.

E 54: Hm, it is the point. So now it intersects line AB. Would it be possible to draw another line, which would not intersect the straight line $p$ ?

Even though in the previous part of the interview Jakub corrected his wrong answer in the right way, the experimenter wants to be sure that the conflict in this context has been really removed and so he asks in a similar way again.

J 55: No.

E 56: Couldn't it be for example this one? (He draws an arch line around the opposite side - see Figure 4.)

J 57: Well, not this one, as the straight line could again be made longer, up to this place (pointing to the picture).

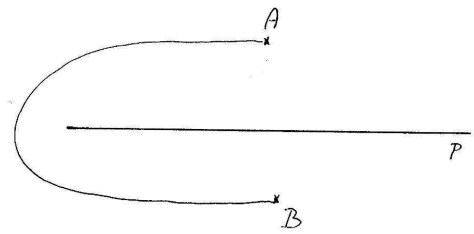

Figure 4

Jakub confirms that he can use the procedure of making the straight line longer securely. The cognitive conflict was induced and then successfully removed. However, we cannot think that the obstacle itself has been overcome. It is possible (and also probable) that it will appear again, this time in a more complex context.

EXAMPLE 2. Michal, school year 9, average student

Attribute of cardinality (distinction of finite, very numerous and infinite sets)

E21: And what about people, all people in the whole world, is their number infinite? 
M22: No, it is not. There was a census of people, flats and households. And these are finite.

E23: And what about fish, all fish worldwide?

M24: Well, this is infinite, it cannot be counted.

E25: And what about (3) ants, ants on the Earth?

M26: The ants on the Earth are infinite.

At this moment, the experimenter noticed a contradiction. Michal uses for the infiniteness of a set the criterion They cannot be counted (understand the elements of the given set), therefore they are infinite. The right or the wrong use of this criterion is lying in the meaning of the statement They can (cannot) be counted. For Michal it means the real realization of the process of counting. This can be proved by statements in positions M22 and M24. Even though the set of all people in the Earth is relatively numerous and he is not able to count them himself, he has the experience that the number of people has already been determined. For "less numerous" sets, the principal and real possibility of counting overlap. Even more, they overlap also for infinite sets (by applying both real and principal counting of the elements of the set the pupil gets the same, i.e. the negative answer). In these contexts, Michal solidifies himself that his knowledge is right. And this knowledge - the understanding the meaning of "it is possible to count it" as a real possibility of counting - is an obstacle for Michal. The experimenter tries to induce a cognitive conflict.

E27: And ants in the Czech Republic?

M28: It's also infinity.

E29: And in one forest?

M30: It's also infinity, still infinity.

E31: And in one ant-hill?

M32: It's also infinity.

Michal perceives the idea of counting the ants in an ant-hill as unreal.

E33: And if I took some ants into a glass, how many of them would be in the glass?

M34: Well, (4) I do not know.

It is possible that the CC has been induced. Michal was aware of the inconsistency of his answers, but it is possible that it has not happened yet.

E35: Is the number of the ants finite or infinite?

M36: Well, finite.

E37: And in one ant-hill?

M38: Well, perhaps also finite. 
"tmcs-eisenmann-etal" — 2009/11/4 — 11:03 — page 289 — \#11

Cognitive conflict as a tool of overcoming obstacles in understanding infinity

Michal starts to reconsider his answers.

E39: And how is it with the ants in the entire world?

M40: They must be also finite.

Michal has changed his previous answer, which leads him to the CC and has corrected it in the right way. It means that in this context he understands a finite set as follows: ,Its elements can be principally counted“. Michal understands that it is not necessary to know the number of set elements but he can imagine the way to determine it. The obstacle has not been totally overcome yet, as it can be seen in the following utterance.

E51: And how many atoms are on the whole Earth?

M52: There is an infinite number of atoms there. (contradiction)

E53: And how many atoms are for example in this nail?

The experimenter tries to induce again the CC. He assumes that Michal would use the analogy from the previous situation.

M54: There is an infinite number as we cannot count them because we cannot even see them.

Michal cannot imagine how to count the atoms, they are unimaginably small, impalpable that they cannot be counted; they are beyond the horizon of discriminability. The idea of counting still prevails in his mind, moved further thanks to the previous experience with the removal of the CC but not the wholly overcoming of it. The ability of children to assess the cardinality of real sets does not relate, in our opinion, directly to age. For example, two years younger Marek has a clear opinion about the question of the cardinality of real sets - about the set of all ants in the world, grains of sand in a desert, or molecules in a room. He says:

It will be a big number, a limited one, but it won't be infinity.

The counting must end somewhere.

The grains of sand in a desert, they are not infinite, it would be possible to count them, it would be really hard, the numbers would be really high, but it would end somewhere ... . Only the number of numbers is infinite.

Marek is sure about this question, he talks about a limited number. He holds out and thinks that the only thing that could be infinite is the number of stars. But he adds Well, maybe.

Marek says: The counting would always end somewhere. In these considerations, he is at such a level that he abstracts away from unreality the process of real counting of set element to the advantage of the principal possibility of counting. 
We dare to raise the hypothesis that the ability of thinking in this way is far less dependent on the age of children than the ability of the right consideration of other attributes of infinity, for example the order density. Here, the creation of right ideas is more connected with school education than with the consideration of set cardinality. During our research, we met A-level grammar school students ${ }^{3}$ who unrelentingly defended their opinions that the number of grains in a sandpit or bugs in the garden is infinite while nine year old children had a clear idea about set cardinality. It can be illustrated by the following sample of an interview between two pupils of school year 3, Jeník and Marie.

EXAMPLE 3. Jeník and Marie, school year 3, very good students

E1: Let's go to another page. What is infinitely many?

M2: Numbers.

J3: Well, yes. There are infinitely many numbers.

E4: Well. (5) And do you think that you would find anything else that is infinite in number? (3) Apart from numbers?

J5: Twaddle. (laugh)

E6: Well, let's try something that we can imagine better. OK? (4) What about (2) people?

J7: No.

E8: All people in the whole world.

M9: No.

J10: There are about five billion of them, so their number cannot be infinite.

E11: Clear. (3) And what about people's hair.

M12: It is not infinite.

E13: No, why?

J14: It must be finite, the number of hairs must end, as the number of people is finite.

\footnotetext{
${ }^{3}$ School system in the Czech Republic: Primary education lasts for nine years divided into two stages of five years (from the age of 6 till the age of 10) and four years respectively (till the age of 15). Secondary education comprises three main types of schools: secondary general schools (grammar school), secondary technical schools and secondary vocational schools. Grammar schools prepare for study at higher education institutions. The secondary technical schools and 4-year courses at secondary vocational schools prepare students for a wide range of professions, as well as for studies at higher education institutions. The 2-year and 3-year courses at the vocational schools prepare students for professional activities.
} 
"tmcs-eisenmann-etal" — 2009/11/4 — 11:03 — page 291 — \#13

E15: Well. (3) And what about something tiny, little, minute. Grains of sand in all deserts and all sandpits.

M16: No, they are not infinite.

J17: No. They must end.

E18: How does it come?

J19: Well, as the desert ends, the grains must end too.

M20: No, if you counted them, you would not count infinity. It would have to end one day, the counting.

EXAMPLE 4. Klára, grammar school year 3, low achiever

Attributes of order (order density), infinite process, convergence

The question "Is it true that $0, \overline{9}<1$ or $0, \overline{9}=1$ ? Explain why.", Klára answers:

K172: When rounded, the number would be equal one, but without rounding it is not the same.

There is a contradiction. We will try to diagnose the obstacle, which is the cause of this contradiction, and we will try to induce a cognitive conflict.

E173: Hm. And why is it not the same?

K174: Zero point nine periodic is not the same as one as it is a bit smaller than one, it is a smaller number.

E175: $H m$.

K176: And it is rounded, we must round it up, we round it to one, but there will be a bit missing.

E177: Well. OK. I will write zero point nine and one. (The experimenter writes on the sheet of paper 0,9 and next to it 1). When you see these next to each other, which of the numbers is smaller?

K178: The 0,9.

E179: Well. And what is the difference between 1 and 0,9?

K180: A tenth.

E181: A tenth. It is a positive number bigger than zero. We can say that one is bigger than 0,9 as the difference is the positive number, it that so?

K182: Hm, yes.

E183: Yes, and if you say that $0, \overline{9}$ is smaller than one, tell me what the difference is between the one and the $0, \overline{9}$.

K184: (2) So there is zero point something, I do not know how much, a millionth, I do not know.

E185: A millionth? 
K186: Well, it is not the one tenth; it is smaller, much smaller.

E187: And what exactly?

K188: (7) Well, there are more $9^{\prime}$ s, it is 0,99, I do not know how far it can go.

The CC has not been induced yet. We expected that it could be induced on position 183, but we can assume that Klára struggled not only with the usual pupils' and students' obstacle If the number starts with 0 , it cannot be 1 , but also with her idea of what the bar above 9 , indicating the period, means.

E189: Well. How many $9^{\prime}$ s are in the number expansion of $0, \overline{9}$ ?

K190: Infinitely many. (2) No, I do not know, there are many of them, plenty of them.

E191: And many, or infinitely many?

K192: (3) I do not know if we can determine their number exactly, or we cannot. E193: Look, Klára, the period means that there is an infinite number of $9^{\prime}$ s and the decimal expansion never ends, you know, the digit 9 recurs for ever.

The experimenter in the following part of the interview, which is not presented here, reminded Klára what the period means and its use in the example with the division of 2 by number 9 . The obstacle of the contradiction presented at the beginning of the interview is also the fact that Klára understands the infinite amount of 9's in the decimal expansion of as naturally infinite, thus it is "extremely big, enormous" (see position K 190).

E302: Well, let's go back to the question whether $0, \overline{9}$ is less than 1 or whether it is equal.

K303: Less, because 0, $\overline{9}$ will never be 1 , as there is still a bit missing.

E304: What bit? The bit between $0, \overline{9}$ and one?

K305: Yes, clear, and we cannot say exactly how big it is.

The presented words prove that Klára does not understand, even after the above mentioned explanation, the decimal extension of $0, \overline{9}$ as infinite. The cognitive conflict was not induced in the intended way (using the difference between 1 and $0, \overline{9}$ ). The experimenter tried to induce the $\mathrm{CC}$ in another way.

E306: Write down, Klára, one divided by nine, next to each other and divide these two numbers. How much is it?

K307: (Dividing on the paper.) Zero point (3), one (4), zero point one periodic.

E308: OK. And two ninths?

K309: (3) Zero point two (2) periodic.

E310: Yes. And three ninths?

K311: Zero point three periodic.

E312: Well, and so on, yes, and eight ninths? 
"tmcs-eisenmann-etal" — 2009/11/4 — 11:03 — page 293 — \#15

K313: Zero point eight periodic.

E314: Hm. And nine ninths?

K315: Zero point nine periodic.

E316: Hm. And but is it possible to express nine ninths in a different way, to express it with different words?

K317: One.

E318: I see. But now you say that one is the same as $0, \overline{9}$.

K319: (2) Well, you are right.

The experimenter succeeded in inducing the CC.

E320: Then, is it the same or not?

K321: (2) Well, so, it is like that.

E322: Like what? What do you mean? Either it is equal to one or it is not, isn't it?

K323: Well, I think that it should not be equal to one as it starts with 0,9 (3), but using the fraction, it equals one.

Klára's obstacle If it starts with zero, it cannot be one, has not been attacked by the above induced CC. We explain this by the fact that the way of inducing the CC does not attack explicitly the obstacles which are its cause. It is in our terminology, the so called vain attempt at removing the conflict: The individual refuses to remove the $\mathrm{CC}$ and the disagreement in his/her knowledge structure endures. Even this version of facing with the CC has its value for him/her, as it is important, from the propedeutic point of view, for other conflicts induced in order to overcome the given obstacle gradually.

\section{Conclusion}

The aim of our research is to specify the process of forming the concept of infinity. This concept is grasped by using other concepts - objects (point, straight line, number etc.) as we learn about its attributes - set cardinality, set boundedness, set measure, ordering, infinite process and convergence. These attributes are in the focus of the structured interview questions.

For the explanation of the process of forming the concept of infinity, we used the theory of epistemological obstacles (Brousseau [1]) that are fundamental for the given concept. Thus we should not avoid obstacles but we should intentionally look for them. It is typical of an obstacle that it resists disagreement with which it is confronted. The key moment for overcoming the obstacle is the inducing and subsequent removal of the cognitive conflict. Only after the removal of many 
"tmcs-eisenmann-etal" — 2009/11/4 — 11:03 — page 294 — \#16

cognitive conflicts in different contexts can we overcome the given obstacle and create "better" pieces of knowledge.

In this article we present four illustrative examples of interviews conducted in the year 2007 with pupils of primary schools and with one grammar school student. The interviews focus on the attributes of infinity - set boundedness and cardinality, ordering (order density), the infinite process and convergence.

The first sample (Jakub, school year 5) demonstrates the inducing of the cognitive conflict, which followed the indicated contradiction caused by an obstacle, i.e. by a replacement of a straight line and its image as a model. The respondent was able to successfully remove the CC.

In the second sample (Michal, school year 9), the interview is focused on the attribute of set cardinality. Also here, the inducing of the $\mathrm{CC}$ was successful after noticing the contradiction by the experimenter. The obstacle is the understanding of the criterion "it is possible to count" as a real possibility of counting. The respondent was successful in removing the induced CC, however only in the given context. In a different context, the respondent fails as the obstacle has not been overcome yet.

The last sample (Klára, grammar school year 3) illustrates the case when first the inducing of the $\mathrm{CC}$ failed as there was a lack of knowledge of the infinite decimal expansion in Klara's knowledge structure. After that the CC was induced in another context, unfortunately with the causing obstacles being attacked. For that reason, it was a vain attempt at $\mathrm{CC}$ removal.

For a successful process of forming the concept of infinity it is necessary to overcome obstacles. It is quite clear from the interviews that the mediator role of the teacher is very important at the building the concept of infinity. In all analyzed cases, the students were not able to develop their conception of infinity until an experimenter's interventions. A teacher needs to know possible obstacles in the learning process. To overcome them gradually, the teacher has to create such didactical situations when cognitive conflicts in different contexts are repeatedly induced and removed. 


$$
\text { "tmcs-eisenmann-etal" — 2009/11/4 — 11:03 — page } 295 \text { — \#17 }
$$

\section{References}

[1] G. Brousseau, Theory of Didactical Situations in Mathematics, (Balacheff, N., ed.), Kluwer Academic Publishers, Dordrecht/Boston/London, 1997.

[2] J. Cihlář, P. Eisenmann, M. Krátká and P. Vopěnka, About a distribution of points on a line segment, in: Mathematics XII, Akademia im. Dlugosza w Czestochowie, Czestochowa, 2007, 175-184.

[3] E. Gray and D. Tall, Duality, ambiguity, and flexibility: A "proceptual" view of simple arithmetic, Journal for Research in Mathematics Education 25, no. 2 (1994), 116-134.

[4] M. Krátká, A Geometrical Picture as an Obstacle, in: Proceeding of SEMT'05, The Charles University, Prague, 2005.

[5] J. Piaget, The Psychology of Intelligence, Routledge and Kegan Paul LTD, London, 1964.

[6] J. Piaget and B. Inhelder, The Psychology of the Child, Routledge and Kegan Paul LTD, London, 1979.

[7] K. Popper, All life is Problem Solving, Routledge, London, 1999.

[8] A. Sierpinska, Understanding in Mathematic, The Falmer Press, London, Washington, D.C., 1994.

[9] M. Swan, Teaching Decimal Place Value, A Comparative Study of Conflict and Positive Only Approaches, University of Nottingham, Shell Centre Nottingham, England, 1983.

[10] D. O. Tall, Conflicts and Catastrophes in the Learning of Mathematics, Math. Education for Teaching 2 (1976), 2-18.

[11] D. O. Tall, Cognitive conflicts and the learning of mathematics, Paper presented at PME 1, Utrecht, Netherlands, 1977.

[12] D. O. Tall and R. L. E. Schwarzenberger, Conflicts in the learning of real numbers and limits, Mathematics Teaching 82 (1978), 44-49.

[13] P. Tsamir, When 'The Same' is not perceived as such: The case of infinite sets, Educational Studies in Mathematics 48 (2001), 289-307.

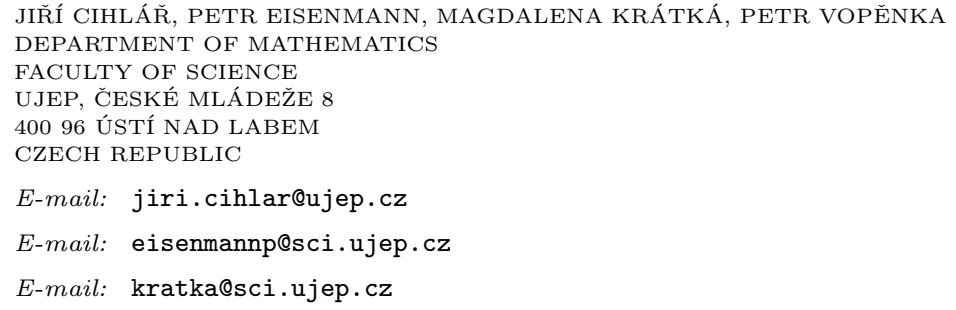

(Received April, 2009) 